\title{
Cholesterol Is Associated with the Presence of a Lipid Core in Carotid Plaque of Asymptomatic, Young-to-Middle-Aged African Americans with and without HIV Infection and Cocaine Use Residing in Inner-City Baltimore, Md., USA
}

\author{
Jiefu Du ${ }^{a}$ Bruce A. Wasserman ${ }^{b}$ Weijing Tong ${ }^{c}$ Shaoguang Chen ${ }^{c}$ \\ Shenghan Lai ${ }^{c}$ Saurabh Malhotrad Hong Lai ${ }^{\text {b }}$ \\ ${ }^{a}$ Department of Emergency Medicine, 301 Hospital, Beijing, China; Departments of badiology and ${ }^{\mathrm{c} P a t h o l o g y,}$ \\ Johns Hopkins School of Medicine, Baltimore, Md., and d Krannert Institute of Cardiology, Indiana University, \\ Indianapolis, Ind., USA
}

\section{Key Words}

Carotid artery $\cdot$ Cholesterol $\cdot$ Lipid core $\cdot$ Risk factors $\cdot$ Stroke

\begin{abstract}
Background: Stroke remains a leading cause of death in the United States. While stroke-related mortality in the USA has declined over the past decades, stroke death rates are still higher for blacks than for whites, even at younger ages. The purpose of this study was to estimate the frequency of a lipid core and explore risk factors for its presence in asymptomatic, young-to-middle-aged urban African American adults recruited from inner-city Baltimore, Md., USA. Methods: Between August 28, 2003, and May 26, 2005, 198 African American participants aged 30-44 years from inner-city Baltimore, Md., were enrolled in an observational study of subclinical atherosclerosis related to HIV and cocaine use. In addition to clinical examinations and laboratory tests, B-mode ultrasound for intima-media thickness of the internal carotid arteries was performed. Among these 198, 52 were selected from the top 30th percentile of maximum carotid intima-media thickness by ultrasound, and high-resolution black blood MRI images were acquired through their carotid plaque before
\end{abstract}

and after the intravenous administration of gadodiamide. Of these 52,37 with maximum segmental thickness by $M R I>1.0$ $\mathrm{mm}$ were included in this study. Lumen and outer wall contours were defined using semiautomated analysis software. The frequency of a lipid core in carotid plaque was estimated and risk factors for lipid core presence were explored using logistic regression analysis. Results: Of the 37 participants in this study, 12 (32.4\%) were women. The mean age was $38.7 \pm$ 4.9 years. A lipid core was present in $9(17 \%)$ of the plaques. Seventy percent of the study participants had a history of cigarette smoking. The mean total cholesterol level was $176.1 \pm$ $37.3 \mathrm{mg} / \mathrm{dl}$, the mean systolic blood pressure was $113.1 \pm 13.3$ $\mathrm{mm} \mathrm{Hg}$, and the mean diastolic blood pressure was $78.9 \pm$ $9.5 \mathrm{~mm} \mathrm{Hg}$. There were 5 participants with hypertension (13.5\%). Twelve (32\%) participants had a history of chronic cocaine use, and 23 (62\%) were HIV positive. Among the factors investigated, including age, sex, blood pressure, cigarette smoking, C-reactive protein, fasting glucose, triglycerides, serum total cholesterol, coronary calcium, cocaine use, and HIV infection, only total cholesterol was significantly associated with the presence of a lipid core. Conclusions: This study revealed an unexpectedly high rate of the presence of lipid core in carotid plaque and highlights the importance of choles-

\section{KARGER}

Fax +41613061234 E-Mail karger@karger.ch www.karger.com

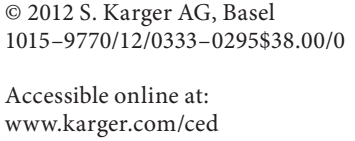

H. Lai, PhD, MPH

Department of Radiology, Johns Hopkins School of Medicine

600 N. Wolfe Street

Baltimore, MD 21287 (USA)

Tel. +1 410955 2216, E-Mail hlail@jhmi.edu 
terol lowering to prevent cerebrovascular disease in this population. Further population-based studies are warranted to confirm these results.

Copyright $\odot 2012$ S. Karger AG, Basel

\section{Introduction}

Black adults not only have the highest coronary artery disease mortality rate of any racial group in the United States $[1,2]$, but they also have a nearly $40 \%$ higher adjusted incidence of ischemic stroke than white adults [3] and higher stroke mortality [4]. African American populations also have a significantly greater common carotid intima-media thickness than non-Hispanic white populations [5-7].

It has been demonstrated that young and middle-aged blacks have a disproportionately high cardiovascular morbidity and mortality $[8,9]$. Although the risk factors for subclinical cerebrovascular disease have been extensively investigated in general, the factors that influence stroke in blacks, especially African American adults residing in an inner-city with lower socioeconomic status, are poorly documented. These factors are even less well understood when the effects of drug use or HIV infection are considered. According to our study, the frequency of the presence of coronary plaque was 30\% in HIV-infected blacks aged 25-54 years; however, the frequency was $60 \%$ in those who used cocaine for longer than 15 years and used antiretroviral medications for more than 18 months [10].

Carotid plaque vulnerability to rupture has been reported to be associated with stroke and other cerebrovascular events [11-13] and a lipid core with a thin fibrous cap that occupies a high proportion of the plaque volume is a predominant indicator of plaque vulnerability [14]. It has been reported that cerebral events can be precipitated by plaque rupture resulting from fibrous cap foam cell infiltration and cap thinning, similar to the mechanism of coronary plaque rupture leading to myocardial events [15]. The objective of this study was to estimate the frequency of a lipid core and explore risk factors for its presence in asymptomatic, African American adults recruited from an inner-city clinic in Baltimore, Md., USA.

\section{Subjects and Methods}

\section{Study Participants}

Between August 28, 2003, and May 26, 2005, 198 African American study participants from inner-city Baltimore were enrolled in an observational study of subclinical atherosclerosis as related to
HIV and cocaine use. The study enrolled African American men and women with or without HIV infection and without cardiovascular diseases. The HIV-positive participants were recruited by flyers posted in and around the Johns Hopkins HIV/AIDS clinic. The remainder were individuals who had also responded to these flyers or were identified by word of mouth or because they were known to others already recruited. Inclusion criteria were age between 25 and 44 years and black race. Exclusion criteria were as follows: (1) any evidence of ischemic heart disease, based on clinical history, previous hospitalization for myocardial infarction, angina pectoris, or electrocardiographic and/or echocardiographic evidence of previous myocardial damage by ischemic heart disease; (2) any symptoms believed to be related to cardiovascular/cerebrovascular disease; (3) pregnancy, and (4) contraindications to MRI with contrast including renal failure. Clinical examinations, electrocardiograms, lipid profiles, high-sensitivity C-reactive protein tests, spiral computed tomographies for coronary calcium, and B-mode ultrasound for intima-media thickness of the internal carotid arteries were performed. Information about sociodemographic characteristics, medical history, medication use, and cocaine use behaviors was obtained by interviewer-administered questionnaires. Among these 198, 52 from the top 30th percentile of maximum carotid intima-media thickness of either the right or left internal carotid artery were selected for the internal carotid artery MRI examinations. The Committee on Human Research at the Johns Hopkins School of Medicine approved the study protocol, and all study participants provided written informed consent. All procedures used in this study were in accordance with institutional guidelines.

\section{Blood Pressure Measurement}

Sitting systolic and diastolic blood pressures were measured twice with a standard mercury sphygmomanometer. A nurse at the clinic measured the study participant's arm circumference and applied a correctly sized cuff. The participant sat quietly for $5 \mathrm{~min}$, and then the nurse obtained the systolic and diastolic blood pressure measurements; a second measurement was made 3 min later. The average of the 2 readings was reported.

Measurement of Lipids

Venous blood samples were obtained after an overnight fast from a large antecubital vein. Serum was separated by centrifugation at $2,000 \mathrm{~g}$ for $15 \mathrm{~min}$ at $4^{\circ} \mathrm{C}$ and stored at $-75^{\circ} \mathrm{C}$ until assayed. Serum lipid parameters, including total cholesterol, triglycerides, high-density lipoprotein cholesterol, and low-density lipoprotein cholesterol were directly determined with the use of a Roche Hitachi 747 analyzer (Englewood, N.J., USA).

The fasting plasma glucose was estimated by the glucose oxidase method (LX20 Synchron Pro; Beckman-Coulter Instruments, Brea, Calif., USA). The diagnosis of diabetes mellitus (fasting plasma glucose $\geq 126 \mathrm{mg} / \mathrm{dl}$ or $7.0 \mathrm{mmol} / \mathrm{l}$ ) was based on the criteria of the American Diabetes Association [16].

\section{MRI Study Protocol}

The MRI study protocol has previously been described in detail [13]. Briefly, MRI examinations were performed on a 1.5-tesla MRI scanner (CV/i scanner; GE Medical Systems, Milwaukee, Wisc., USA) using a dual 3 -inch $(7.6 \mathrm{~cm})$ receive-only carotid surface coil fixed over each side of the neck.

A 3-dimensional time-of-flight MR angiogram was acquired to localize the carotid bifurcations. Three proton-density-weight- 
ed black blood images were oriented through the long axis of the bifurcation on the side selected based on thickness determined by ultrasound in advance of the MRI examinations. These images were used to position $5 \mathrm{~T}_{1}$ - and $\mathrm{T}_{2}$-weighted fat-suppressed black blood images perpendicular to the area of greatest wall thickness with the middle slice traversing the most stenotic point. Black blood images were acquired using a double inversion recovery fast spin echo sequence with peripheral pulse gating. The acquired resolution was $2 \times 0.54 \times 0.54 \mathrm{~mm}$ [17].

Participants received an intravenous injection of $0.1 \mathrm{mmol} / \mathrm{kg}$ gadopentetate dimeglumine (Magnevist; Bayer Healthcare) at a rate of $2 \mathrm{ml} / \mathrm{s}$ through a power injector.

The $5 \mathrm{~T}_{1}$-weighted transverse black blood images were repeated 5 min after gadolinium administration with the inversion time adjusted to suppress the signal of flowing blood [17].

\section{MRI Analysis}

The postcontrast transverse image showing the largest lipid core area, or the thickest wall if no core was present, was analyzed using Vesselmass software (VesselMass; Division of Image Processing, Radiology Department, Leiden University Medical Center). An analyst drew lumen, lipid core (if present), and outer wall contours on this image using the software. Contours were reviewed by an experienced MRI physician. Observers were blinded to characteristics of the study population. The postcontrast image was used based on reports that gadolinium enhancement improves delineation of the lipid core and outer wall [18]. The corresponding $\mathrm{T}_{2}$-weighted image was used to confirm lipid core delineation when questionable on the postcontrast series, and the corresponding precontrast $\mathrm{T}_{1}$-weighted image was used to confirm the lumen contour when flow artifact from gadolinium was present [17]. The vessel wall was divided into 12 radial segments by a semiautomated feature of Vesselmass software.

\section{Statistical Analysis}

We restricted our analysis for lipid core presence to those participants whose maximum wall thickness was $\geq 1.0 \mathrm{~mm}$ to ensure that small cores were not missed because of the resolution constraints of the MRI technique. No lipid cores were excluded by this restriction. SAS software (version 9.1) was used for all analyses. Categorical variables are presented as numbers and percentages, and were compared using the $\chi^{2}$ test. Continuous variables are expressed as mean $\pm \mathrm{SD}$, and were compared using Student's test. Univariate logistic regression models were first fitted to evaluate the crude association between the presence of a lipid core and each of the factors, including age, sex, HIV infection, total serum cholesterol, high-density lipoprotein cholesterol, low-density lipoprotein cholesterol, serum triglycerides, high-sensitivity C-reactive protein, cigarette smoking, alcohol use, glucose level, blood pressure, body mass index, cocaine or other illicit drug (opiates, benzodiazepines, methamphetamine) or alcohol use, and coronary calcium score, individually. Those factors that were significant at the $\mathrm{p} \leq 0.10$ level in the univariate models were put into the multiple logistic regression models to identify the factors that were independently associated with the presence of lipid core. Those variables that ceased to make significant contributions to the models were deleted in a stagewise manner, yielding the final models. A significance level was set at $\mathrm{p}<0.05$ and all $\mathrm{p}$ values reported were two-sided.

Lipid Core in Carotid Plaque of Blacks

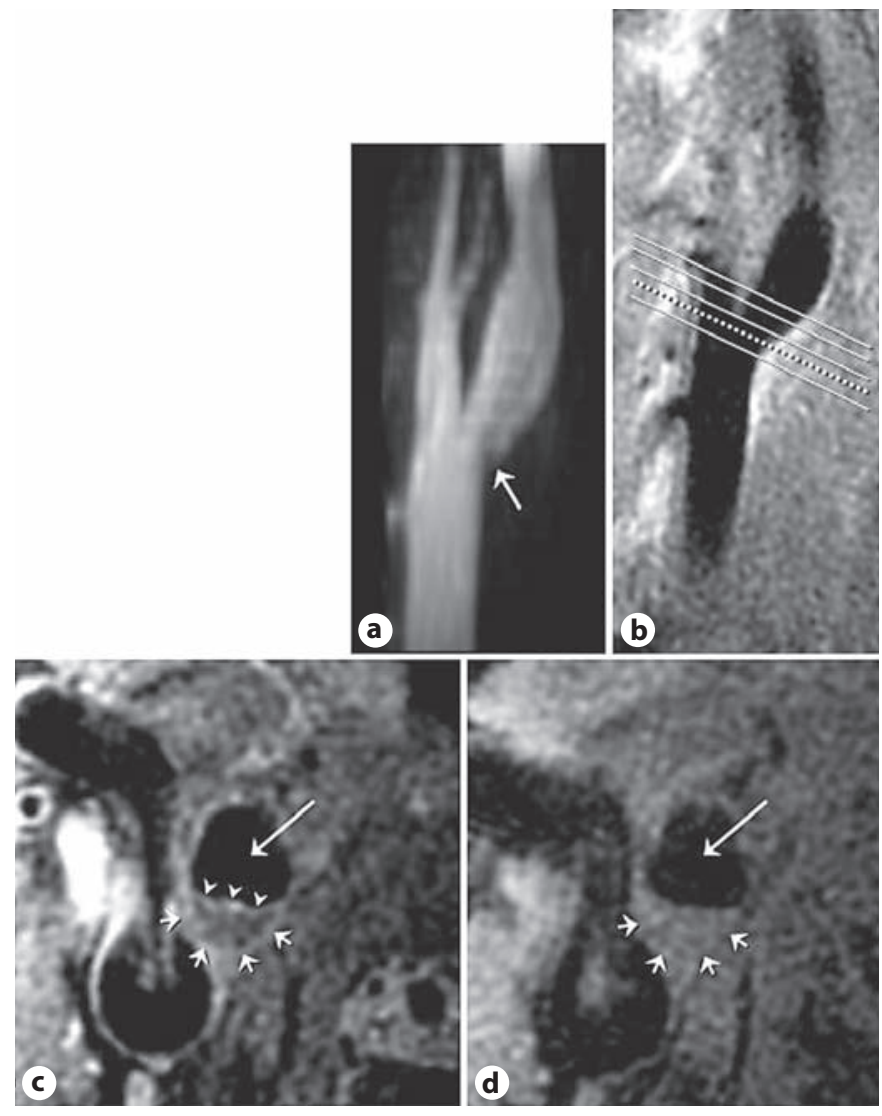

Fig. 1. MRI images of the right carotid bifurcation in a 46-year-old female without HIV infection or cocaine use. a MR angiogram maximum intensity projection image demonstrates slight indentation into the base of the carotid bulb by an atherosclerotic plaque (arrow). b A long-axis black blood MRI image is used to orient 5 slices through the plaque (lines) along the carotid bulb. c A short-axis postcontrast black blood MRI image oriented through the plaque seen on the long-axis image (dotted line, b) demonstrates the eccentric lesion (short arrows) with an enhancing fibrous cap (arrowheads) separating the lumen (long arrow) from the lipid core (central hypointense tissue). d A precontrast image shows the eccentric plaque (short arrows) and lumen (long arrow) but a relatively inconspicuous core.

\section{Results}

\section{General Characteristics}

Of the above-mentioned 52 participants, 15 were excluded because their maximum segmental thickness by MRI was $<1.0 \mathrm{~mm}$. The remaining 37 participants had a mean maximum segmental thickness measurement of $1.60 \pm 0.81 \mathrm{~mm}$. Two sets of MRI images are presented (fig. 1,2). Figure 1 depicts MRI images of a plaque with a lipid core and figure 2 shows a plaque without a lipid core (a simple plaque). 


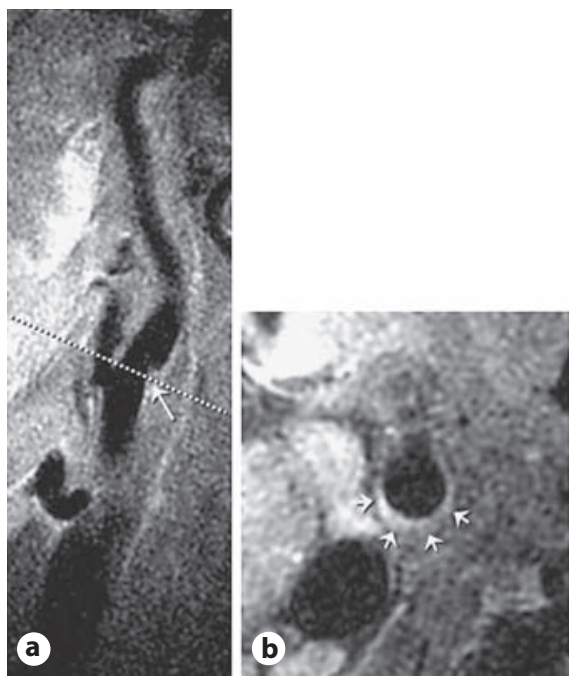

Fig. 2. Black blood MRI images of the right carotid bifurcation in a 33 -year-old female with HIV infection and cocaine use. Longaxis view used to orient short-axis images shows a focal plaque (arrow) along the outer wall of the carotid bulb. The short-axis view (b) through the plaque (line, a) shows the eccentric wall thickening (arrows, b).

Demographic and clinical characteristics of the study participants are presented in table 1 . Of the 37 participants in this study, $12(32.4 \%)$ were women. The mean age was $38.7 \pm 4.9$ years. Seventy percent of the study participants had a history of cigarette smoking. The mean total cholesterol level was $176.1 \pm 37.3 \mathrm{mg} / \mathrm{dl}$, the mean systolic blood pressure was $113.1 \pm 13.3 \mathrm{~mm} \mathrm{Hg}$, and the mean diastolic blood pressure was $78.9 \pm 9.5$ $\mathrm{mm} \mathrm{Hg}$. There were 5 participants with hypertension (13.5\%). Thirty percent had a positive coronary artery calcium score. Among the 37 study participants, 23 (62.2\%) were HIV-infected, and 12 (32.4\%) were chronic cocaine users.

\section{Frequency of and Associations with the Presence of}

Lipid Core in Carotid Plaque

Of the 37 study participants, $9(24.3 \%, 95 \% \mathrm{CI}=0.12$ $0.41)$ had a lipid core on the postcontrast MRI image. The frequencies of the presence of lipid core were $0.0 \%(0 / 10)$, $33.3 \%(3 / 9)$, and $33.3 \%(6 / 18)$ in those whose age was less than or equal to 35 years, above 35 but less or equal to 40 years, and above 40 years, respectively. The frequencies of the presence of lipid core were $24.0 \%(6 / 25)$ in males and $25.0 \%(3 / 12)$ in females.
Demographic characteristics and MRI measurements by the presence of a lipid core are presented in tables 1 and 2 , respectively. As shown in table 1, among the variables investigated, only total cholesterol level was significantly associated with the presence of lipid core $(p=0.04)$ according to the $t$ test. Compared with those without lipid core, those with lipid core had a significantly higher mean wall thickness $(\mathrm{p}=0.006)$ and maximum wall thickness $(\mathrm{p}=0.003)$.

According to univariate logistic regression analyses, only total cholesterol level in log scale $(\mathrm{p}=0.049)$ was significantly associated with the presence of lipid core. Thus, the final multiple logistic regression analysis revealed that only total cholesterol level (log scale) was independently associated with the presence of lipid core. Age, sex, cigarette smoking, body mass index, triglycerides, highsensitivity C-reactive protein, blood pressure, coronary artery calcification, HIV infection, and cocaine use were not significantly associated with the presence of lipid core (table 3).

\section{Discussion}

To our knowledge, this is the first study to estimate the frequency of the presence of lipid core in carotid plaque in asymptomatic, young-to-middle-aged African American adults recruited from an inner-city clinic.

A high frequency (24\%) of a lipid core in carotid plaque in this group aged 35 or older is quite disturbing. Although black adults have a higher stroke mortality than white adults, the underlying mechanisms remain poorly understood. The higher frequencies of hypertension and diabetes have been associated with the excess stroke mortality $[19,20]$. Furthermore, a lower socioeconomic status is associated with increased risk of stroke [21].

The MESA (Multi-Ethnic Study of Atherosclerosis) study has demonstrated that in those aged between 45 and 84 years with thickened carotid walls, plasma total cholesterol is independently associated with lipid core presence by MRI [17]. Our study in much younger African American adults from the inner city also demonstrates that elevated total cholesterol levels, not other coronary artery disease risk factors, were a strong and independent predictor of the presence of lipid core with the use of high-resolution MRI. Thus, our findings can be considered evidence that blood cholesterol levels may be of prime importance for the development of the clinically important lipid-rich atherosclerotic plaque. Actually, accumulation of cholesterol plays a central role in athero- 
Table 1. Characteristics of study participants, by the presence of a lipid core

\begin{tabular}{|c|c|c|c|c|}
\hline Variable & $\begin{array}{l}\text { All } \\
(\mathrm{n}=37)\end{array}$ & $\begin{array}{l}\text { Without lipid core } \\
(\mathrm{n}=28)\end{array}$ & $\begin{array}{l}\text { With lipid core } \\
(n=9)\end{array}$ & $\begin{array}{l}\mathrm{p} \\
\text { value }^{\mathrm{a}}\end{array}$ \\
\hline Age, years & $38.7 \pm 4.9$ & $38.1 \pm 5.2$ & $40.6 \pm 3.2$ & 0.18 \\
\hline \multicolumn{5}{|l|}{ Age group } \\
\hline $30-35$ & 27.0 & 35.7 & 0 & \multirow[t]{3}{*}{0.11} \\
\hline $35.1-40$ & 24.3 & 21.4 & 33.3 & \\
\hline $40.1-44$ & 48.7 & 42.9 & 66.7 & \\
\hline Female & 32.4 & 32.1 & 33.3 & 1.00 \\
\hline Family history of stroke & 12.1 & 8.0 & 25.0 & 0.24 \\
\hline Family history of heart attack & 22.9 & 19.2 & 33.3 & 0.40 \\
\hline Ever cigarette smoking & 70.3 & 71.4 & 66.7 & 1.00 \\
\hline Ever alcohol consumption & 73.5 & 72.0 & 77.8 & 1.00 \\
\hline HIV infection & $23(62.2)$ & 64.3 & 55.6 & 0.70 \\
\hline Cocaine use $\geq 15$ years & 32.4 & 32.1 & 33.3 & 1.00 \\
\hline Ever cocaine use & 67.6 & 64.3 & 77.8 & 0.69 \\
\hline Body mass index & $25.4 \pm 4.4$ & $25.8 \pm 4.5$ & $24.2 \pm 4.0$ & 0.35 \\
\hline High-sensitivity CRP, mg/l & $4.6 \pm 12.5$ & $5.1 \pm 14.1$ & $3.2 \pm 5.9$ & 0.57 \\
\hline Systolic blood pressure, $\mathrm{mm} \mathrm{Hg}$ & $113.1 \pm 13.3$ & $115.0 \pm 13.5$ & $107.4 \pm 11.3$ & 0.14 \\
\hline Hypertension & $5(13.5)$ & $5(17.9)$ & 0 & 0.17 \\
\hline Diastolic blood pressure, $\mathrm{mm} \mathrm{Hg}$ & $78.9 \pm 9.5$ & $79.1 \pm 10.4$ & $78.3 \pm 6.5$ & 0.84 \\
\hline Fasting glucose, $\mathrm{mg} / \mathrm{dl}$ & $106.9 \pm 85.9$ & $113.3 \pm 94.7$ & $79.0 \pm 8.7$ & 0.22 \\
\hline Total cholesterol, mg/dl & $176.1 \pm 37.3$ & $169.0 \pm 35.0$ & $198.1 \pm 37.7$ & 0.04 \\
\hline HDL cholesterol, mg/dl & $54.2 \pm 16.8$ & $52.2 \pm 14.5$ & $60.3 \pm 22.4$ & 0.21 \\
\hline LDL cholesterol, mg/dl & $103.6 \pm 33.5$ & $98.8 \pm 32.4$ & $118.3 \pm 34.6$ & 0.13 \\
\hline Triglycerides, mg/dl & $100.8 \pm 75.4$ & $102.3 \pm 82.2$ & $96.3 \pm 52.4$ & 0.84 \\
\hline Coronary artery calcification & 29.7 & 32.1 & 22.2 & 0.69 \\
\hline
\end{tabular}

Figures are means $\pm \mathrm{SD}$ or numbers with percentages in parentheses. $\mathrm{CRP}=\mathrm{C}$-reactive protein; $\mathrm{HDL}=$ high-density lipoprotein; $\mathrm{LDL}=$ low-density lipoprotein. ${ }^{\mathrm{a}} \mathrm{p}$ value was for the difference between without and with a lipid core.

Table 2. MRI measurements of study participants, by the presence of a lipid core

\begin{tabular}{|c|c|c|c|c|}
\hline Variable & $\begin{array}{l}\text { All } \\
(n=37)\end{array}$ & $\begin{array}{l}\text { Without lipid } \\
\text { core }(\mathrm{n}=28)\end{array}$ & $\begin{array}{l}\text { With lipid } \\
\text { core }(n=9)\end{array}$ & $\mathrm{p}$ value \\
\hline Mean wall thickness, mm & $1.04 \pm 0.27$ & $0.94 \pm 0.15$ & $1.34 \pm 0.33$ & 0.006 \\
\hline Maximum wall thickness, $\mathrm{mm}$ & $1.60 \pm 0.81$ & $1.25 \pm 0.27$ & $2.67 \pm 0.99$ & 0.003 \\
\hline Area of lumen, $\mathrm{mm}^{2}$ & $0.60 \pm 0.22$ & $0.63 \pm 0.23$ & $0.54 \pm 0.19$ & 0.29 \\
\hline Area of outer, $\mathrm{mm}^{2}$ & $0.92 \pm 0.31$ & $0.92 \pm 0.31$ & $0.94 \pm 0.32$ & 0.84 \\
\hline Lumen/outer ratio & $0.65 \pm 0.07$ & $0.68 \pm 0.04$ & $0.57 \pm 0.08$ & 0.002 \\
\hline
\end{tabular}

genesis [22]. This investigation may be the first study to explore the relations between plaque lipid core detected by MRI and the major cardiovascular risk factors measured in asymptomatic, young African Americans living in an inner city.

The results of our study showed that among all the potential risk factors, including age, sex, family history of stroke, cigarette smoking, systolic blood pressure, dia- stolic blood pressure, high-sensitivity C-reactive protein, body mass index, fasting glucose, triglycerides, high-density lipoprotein cholesterol, low-density lipoprotein cholesterol, and total cholesterol, only total cholesterol was independently associated with the presence of lipid core, as also reported in the MESA study [17].

This study has several limitations. First, the study participants were a convenience sample of a very select- 
Table 3. Associations of demographic and cardiovascular risk factors with the presence of lipid core: logistic regression analyses

\begin{tabular}{|c|c|c|c|c|}
\hline \multirow[t]{2}{*}{ Variable } & \multicolumn{2}{|l|}{ Univariate model } & \multicolumn{2}{|l|}{ Final model } \\
\hline & odds ratio $(95 \% \mathrm{CI})$ & $\mathrm{p}$ value & odds ratio $(95 \% \mathrm{CI})$ & $\mathrm{p}$ value \\
\hline Age & $1.13(0.94-1.36)$ & 0.18 & & \\
\hline Female sex & $1.06(0.21-5.21)$ & 0.95 & & \\
\hline Family history of stroke & $3.83(0.44-33.11)$ & 0.22 & & \\
\hline Family history of heart attack & $2.1(0.39-11.43)$ & 0.39 & & \\
\hline Cigarette smoking & $0.8(0.16-4)$ & 0.79 & & \\
\hline Alcohol consumption & $1.36(0.23-8.21)$ & 0.74 & & \\
\hline HIV infection & $0.69(0.15-3.19)$ & 0.64 & & \\
\hline Cocaine use $\geq 15$ years & $1.06(0.21-5.21)$ & 0.95 & & \\
\hline Cocaine use & $1.94(0.34-11.2)$ & 0.46 & & \\
\hline Body mass index & $0.91(0.75-1.1)$ & 0.34 & & \\
\hline High-sensitivity CRP & $0.98(0.9-1.07)$ & 0.7 & & \\
\hline Systolic blood pressure & $0.95(0.89-1.02)$ & 0.15 & & \\
\hline Diastolic blood pressure & $0.99(0.92-1.07)$ & 0.84 & & \\
\hline Fasting glucose & $0.94(0.82-1.07)$ & 0.35 & & \\
\hline Total cholesterol ${ }^{\mathrm{a}}$ & $73.8(1.02$ to $>999)$ & 0.049 & $73.8(1.02$ to $>999)$ & 0.049 \\
\hline HDL $_{\text {cholesterol }}{ }^{\mathrm{a}}$ & $4.3(0.31-59.7)$ & 0.26 & & \\
\hline LDL cholesterol $^{\mathrm{a}}$ & $7.85(0.56-111)$ & 0.13 & & \\
\hline Triglycerides & $1(0.99-1.01)$ & 0.84 & & \\
\hline Coronary artery calcification & $0.6(0.1-3.51)$ & 0.57 & & \\
\hline
\end{tabular}

$\mathrm{CRP}=\mathrm{C}$-reactive protein; $\mathrm{HDL}=$ high-density lipoprotein; $\mathrm{LDL}=$ low-density lipoprotein. ${ }^{\mathrm{a}}$ In log scale.

ed population. Thus, the results must be interpreted with caution. Second, since the majority of participants were current smokers, the effects of cigarette smoking on the frequency of the presence of lipid core could not be evaluated, either individually or in combination. Third, the sample size of this study was relatively small due to limited resources. Fourth, owing to the nature of the cross-sectional design, some hidden confounding factors, such as socioeconomic status, were not adjusted for. Fifth, we did not collect any data on statin use and could not evaluate whether stain use would retard the lipid core. Furthermore, since this study was performed in African Americans living in inner-city Baltimore, some hidden risk factors in relation to the presence of lipid core could not be completely controlled for by statistical analyses.

Despite its limitations, this study's findings of an unexpectedly high rate of the presence of lipid core in carotid plaque in this asymptomatic, young-to-middle-aged African American adults have disturbing but important implications for the early prevention of cerebrovascular disease in this population. Future large studies confirming these findings that can be generalized to an urban, African American population are warranted.

\section{Disclosure Statement}

The authors declare that they have no competing interests.

\section{Acknowledgments}

The study was supported by grants from the National Institute on Drug Abuse, National Institutes of Health (NIH R01DA 12777 and DA25524).

References

$\mathrm{Du} /$ Wasserman/Tong/Chen/Lai/ Malhotra/Lai 
5 D’Agostino RB Jr, Burke G, O’Leary D, et al: Ethnic differences in carotid wall thickness: the Insulin Resistance Atherosclerosis Study. Stroke 1996;27:1744-1749.

$\checkmark 6$ Manolio TA, Burke GL, Psaty BM, et al: Black-white differences in subclinical cardiovascular disease among older adults: the Cardiovascular Health Study. CHS Collaborative Research Group. J Clin Epidemiol 1995;48:1141-1152.

7 Pasternak RC, Criqui MH, Benjamin EJ, Fowkes FG, Isselbacher EM, McCullough PA, Wolf PA, Zheng ZJ, American Heart Association: Atherosclerotic Vascular Disease Conference: writing group I: epidemiology. Circulation 2004;109:2605-2612.

$>8$ Geronimus AT, Bound J, Waidmann TA, Hillemeier MM, Burns PB: Excess mortality among blacks and whites in the United States. N Engl J Med 1996;21;335:1552-1558.

-9 Bibbins-Domingo K, Pletcher MJ, Lin F, Vittinghoff E, Gardin JM, Arynchyn A, Lewis CE, Williams OD, Hulley SB: Racial differences in incident heart failure among young adults. N Engl J Med 2009;360:1179-1190.

10 Lai S, Bartlett J, Lai H, Moore R, Cofrancesco J Jr, Pannu H, Tong W, Meng W, Sun H, Fishman EK: Long-term combination antiretroviral therapy is associated with the risk of coronary plaques in African Americans with HIV infection. AIDS Patient Care STDS 2009;23:815-824.
11 Yamada K, Yoshimura S, Kawasaki M, Enomoto Y, Asano T, Minatoguchi S, Iwama T: Effects of atorvastatin on carotid atherosclerotic plaques: a randomized trial for quantitative tissue characterization of carotid atherosclerotic plaques with integrated backscatter ultrasound. Cerebrovasc Dis 2009;28: 417-424.

12 Polak JF, Shemanski L, O’Leary DH, Lefkowitz D, Price TR, Savage PJ, Brant WE, Reid C: Hypoechoic plaque at US of the carotid artery: an independent risk factor for incident stroke in adults aged 65 years or older. The Cardiovascular Health Study. Radiology 1998;208:649-654.

$>13$ Gronholdt ML, Nordestgaard BG, Schroeder TV, Vorstrup S, Sillesen H: Ultrasonic echolucent carotid plaques predict future strokes. Circulation 2001;104:68-73.

14 Kiousis DE, Rubinigg SF, Auer M, Holzapfel GA: A methodology to analyze changes in lipid core and calcification onto fibrous cap vulnerability: the human atherosclerotic carotid bifurcation as an illustratory example. J Biomech Eng 2009;131:121002.

15 Carr S, Farb A, Pearce WH, Virmani R, Yao JS: Atherosclerotic plaque rupture in symptomatic carotid artery stenosis. J Vasc Surg 1996;23:755-765.

16 American Diabetes Association: Diagnosis and classification of diabetes mellitus. Diabetes Care 2004;27(suppl 1):S5-S10.
17 Wasserman BA, Sharrett AR, Lai S, Gomes AS, Cushman M, Folsom AR, Bild DE, Kronmal RA, Sinha S, Bluemke DA: Risk factor associations with the presence of a lipid core in carotid plaque of asymptomatic individuals using high-resolution MRI: the multiethnic study of atherosclerosis (MESA). Stroke 2008;39:329-335.

18 Wasserman BA, Smith WI, Trout HH 3rd, Cannon RO 3rd, Balaban RS, Arai AE: Carotid artery atherosclerosis: in vivo morphologic characterization with gadolinium-enhanced double-oblique MR imaging initial results. Radiology 2002;223:566-573.

19 Kittner SJ, White LR, Losonczy KG, Wolf PA, Hebel JR: Black-white differences in stroke incidence in a national sample. The contribution of hypertension and diabetes mellitus. JAMA 1990;264:1267-1270.

20 Gillum RF: Stroke mortality in blacks. Disturbing trends. Stroke 1999;30:1711-1715.

21 Kleindorfer DO, Lindsell C, Broderick J, Flaherty ML, Woo D, Alwell K, Moomaw CJ, Ewing I, Schneider A, Kissela BM: Impact of socioeconomic status on stroke incidence: a population-based study. Ann Neurol 2006; 60:480-484.

22 Badimon JJ, Ibanez B, Cimmino G: Genesis and dynamics of atherosclerotic lesions: implications for early detection. Cerebrovasc Dis 2009;27(suppl 1):38-47. 